\title{
Single center retrospective analysis of preoperative fibrinogen and transfusional requirements in cardiac surgery patients
}

Antunes P., Muchacho P., Santos J., Pedrosa F., Sousa L., Pires I.

Centro Hospitalar de Lisboa Norte - Hospital de Santa Maria, Dept of Anaesthesiology \& Pain Medicine, Lisbon, Portugal

\section{BACKGROUND AND GOAL OF THE STUDY}

Fibrinogen is a key factor in the coagulation cascade.

We examined the association between preoperative fibrinogen levels and red blood cell transfusion in patients submitted to off-pump coronary artery bypass graft surgery in a University Hospital in Portugal.

\section{MATERIALS AND METHODS}

Single center retrospective analysis, with Local Ethics Commitee approval. A random sample of records from $40 \%$ of the patients submitted to off-pump coronary artery bypass graft surgery in 2013 were assessed.

Inclusion criteria: patients without known coagulation disorders.

Statistic analysis: SPSS ${ }^{\circledR}$ V21.0 (Chi-square and Kruskal-Wallis tests; a simple binary logistic regression was performed to identify independent factors for transfusion; $p<0.05)$.

RESULTS Random sample $(\mathrm{n}=132) \longrightarrow$ Missing records $(\mathrm{n}=53) \mathrm{Study}$ group $(\mathrm{n}=79)$

Characteristics related to preoperative fibrinogen concentration

\begin{tabular}{|c|c|c|c|c|c|}
\hline \multirow[b]{3}{*}{ Variables } & \multicolumn{5}{|c|}{ Fibrinogen concentration mg/dL } \\
\hline & & $1^{\circ}$ tertile & $2^{\circ}$ tertile & $3^{\circ}$ tertile & \\
\hline & Total $(n=79)$ & $223-293(n=26)$ & $294-367(n=27)$ & $368-606(n=26)$ & $p$ value \\
\hline Age (years) & $66.47 \pm 9.41$ & $63.81 \pm 7,7$ & $66.81 \pm 8.6$ & $68.77 \pm 11,2$ & 0,04 \\
\hline Gender $(\hat{O}: \uparrow)$ & 62 (78.5):17 (21.5) & 17 (65.4): 9 (34.6) & 23 (85.29): 4 (14.8) & 22 (84.6): 17 (21.5) & 0,140 \\
\hline $\mathrm{BMI}\left(\mathrm{kg} / \mathrm{m}^{2}\right)$ & $28.05 \pm 6.5$ & $27.3 \pm 3.0$ & $27.6 \pm 5.3$ & $29.1 \pm 9.7$ & 0,972 \\
\hline Aspirin & $42(53.2)$ & $15(57.7)$ & $12(44.4)$ & $15(57.7)$ & 0,535 \\
\hline Clopidogrel (with or without aspirin) & $20(25.3)$ & $4(15.4)$ & 9 (33.3) & 7 (26.9) & 0,315 \\
\hline \multicolumn{6}{|l|}{ Preoperative: } \\
\hline Hemoglobin (g/dL) & $13,86 \pm 1,43$ & $14.0 \pm 1.6$ & $14.08 \pm 1.3$ & $13.5 \pm 1.2$ & 0,338 \\
\hline Platelet count (x109/L) & $233,15 \pm 79,8$ & $212,3 \pm 79,4$ & $214,15 \pm 52,3$ & $273 \pm 90.3$ & 0,006 \\
\hline Fibrinogen (mg/dL) & $340,6 \pm 78,9$ & $259.8 \pm 20.8$ & $333.41 \pm 20.6$ & $428.73 \pm 60.5$ & $<0,001$ \\
\hline INR & $0.98 \pm 0.07$ & $0.99 \pm 0.06$ & $0.96 \pm 0.05$ & $0.98 \pm 0.05$ & 0,430 \\
\hline aPTT (s) & $31.82 \pm 3.6$ & $31.2 \pm 3.6$ & $31.9 \pm 3.3$ & $32.5 \pm 3.8$ & 0,355 \\
\hline Number of grafts & $2,16 \pm 0,7$ & $2,19 \pm 0.8$ & $2.22 \pm 0.6$ & $2.08 \pm 0,7$ & 0,637 \\
\hline Tranexamic acid (yes) & $61(77.2)$ & $20(76.9)$ & $21(77.8)$ & $20(76.9)$ & 0,996 \\
\hline Duration of surgery (minutes) & $137,84 \pm 40,08$ & $140,5 \pm 42,3$ & $139,4 \pm 36.8$ & $133,5 \pm 42,1$ & 0,757 \\
\hline Postoperative hemoglobin (g/dL) & $10,42 \pm 2,38$ & $10.8 \pm 1,6$ & $10,7 \pm 1.7$ & $9.6 \pm 3.2$ & 0,248 \\
\hline Duration of hospitalization (days) & $14,53 \pm 16,31$ & $11.2 \pm 16.9$ & $15.11 \pm 15.9$ & $17.2 \pm 16,3$ & 0,026 \\
\hline
\end{tabular}

Data are expressed as the mean \pm SD or number (\%). aPTT, activated partial thromboplastin time, BMI, Body Mass Index; INR, International normalized ratio;

Proportion of patients receiving transfusion of packed red blood cells

60

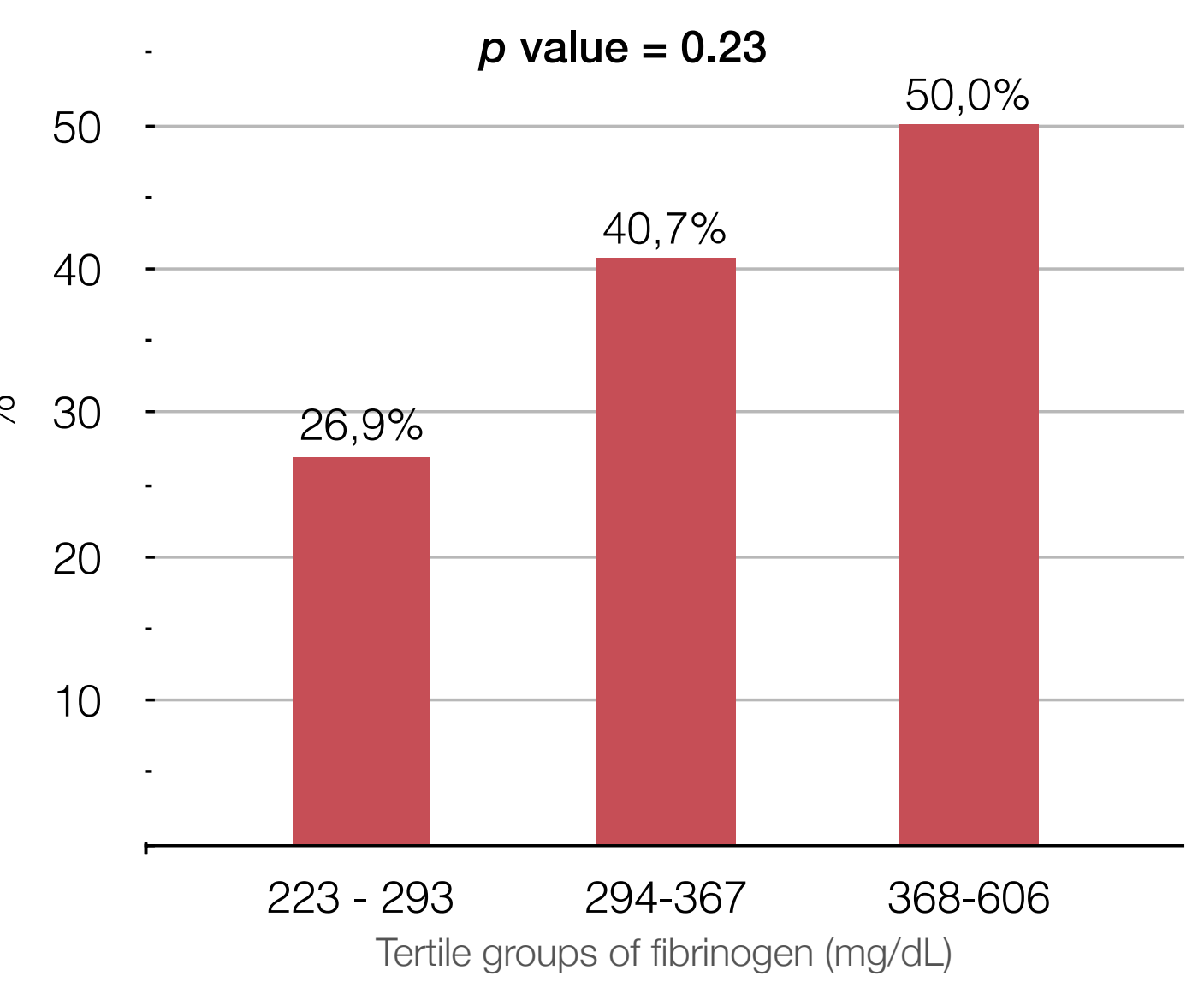

Simple binary logistic regression - Independent risk factors for red blood cell transfusion

\begin{tabular}{|c|c|c|}
\hline Variable & OR (Cl 95\%) & $p$ value \\
\hline Age & $1.1(1.00-1.12)$ & 0,028 \\
\hline Gender $\left(\uparrow: \bigcirc^{\top}\right)$ & 3.85 (1.24-11.89) & 0,019 \\
\hline
\end{tabular}

\section{CONCLUSION}

Although not statistically significant we observed a tendency for higher transfusion rates in groups with higher fibrinogen levels. Age and female gender are independent risk factors for red blood cells transfusion. 